\title{
Tratamento da depressão
}

\author{
Fábio Gomes de Matos e Souza ${ }^{1}$
}

\section{Resumo}

O tratamento antidepressivo deve ser realizado considerando os aspectos biológicos, psicológicos e sociais do paciente. Na média, não há diferenças significativas em termos de eficácia entre os diferentes antidepressivos mas o perfil em termos de efeitos colaterais, preço, risco de suicídio, tolerabilidade varia bastante o que implica em diferenças na efetividade das drogas para cada paciente. A conduta, portanto, deve ser individualizada. A prescrição profilática de antidepressivos irá depender da intensidade e frequiência dos episódios depressivos. O risco de suicídio dever ser sempre avaliado e se necessário o ECT deverá ser indicado. Não há antidepressivo ideal, entretanto, atualmente existe uma disponibilidade grande de drogas atuando através de diferentes mecanismos de ação o que permite que, mesmo em depressões consideradas resistentes, o tratamento possa obter êxito.

\section{Descritores}

Depressão; tratamento; profilaxia; antidepressivos; ECT

\begin{abstract}
The antidepressant treatment should be accomplished considering the patient's biological, psychological and social aspects. In average, there are no significant differences in terms of efficacy among the different antidepressants but the profile in terms of collateral effects, price, suicide risk, tolerability shows great variation which implies in differences in the effectiveness of drugs for each patient. The therapy, therefore, should be individualized. The prophylactic prescription of antidepressants will depend on the intensity and frequency of the depressive episodes. The suicide risk should always be evaluated and if necessary ECT should be prescribed. There is no ideal antidepressant, however, there is a large variety of drugs acting through different mechanisms of action which allows the possibility of therapeutic success in resistant depressions.
\end{abstract}

\section{Keywords}

Depression; treatment; prophilaxis; antidepressants; ECT

\section{Introdução}

O moderno tratamento da depressão apresenta uma gama de opções que permitirá uma flexibilidade ao psiquiatra clínico, no sentido de adequar para cada paciente a melhor abordagem terapêutica. Esta revisão discutirá os pontos mais importantes da terapia antidepressiva, adotando a forma de perguntas e respostas. Outras revisões devem ser mencionadas. ${ }^{1-14}$

\section{Discussão}

Qual deve ser a participação do paciente no tratamento da depressão?

O tratamento deve ser realizado COM o paciente e não PARA o paciente. Assim, o médico deverá disponibilizar para o paciente informações concernentes à depressão bem como às opções terapêuticas.

\section{Como tratar a depressão?}

O tratamento antidepressivo deve ser entendido de uma forma globalizada levando em consideração o ser humano como um todo incluindo dimensões biológicas, psicológicas e sociais. ${ }^{4}$ Portanto, a terapia deve abranger todos esses pontos e utilizar a psicoterapia, mudanças no estilo de vida e a terapia farmacológica. Apesar de o enfoque desta revisão se concentrar na psicofarmaterapia, deve-se mencionar que não se trata "depressão" de forma abstrata mas sim pacientes deprimidos, contextualizados em seus meios sociais e culturais e compreendidos nas suas dimensões biológicas e psicológicas.

As intervenções psicoterápicas podem ser de diferentes formatos, como psicoterapia de apoio, psicodinâmica breve, terapia interpessoal, comportamental, cognitiva comportamental, de grupo, de casais e de família. Fatores que influenciam no sucesso psicoterápico incluem: motivação, depressão leve ou moderada, ambiente estável e capacidade para insight. ${ }^{2}$

Mudanças no estilo de vida deverão ser debatidas com cada paciente, objetivando uma melhor qualidade de vida.

Os antidepressivos produzem, em média, uma melhora dos sintomas depressivos de $60 \%$ a $70 \%$, no prazo de um mês, enquanto a taxa de placebo é em torno de $30 \% .^{15-17}$

Esta taxa de melhora dificilmente é encontrada em outras abordagens terapêuticas de depressão, a não ser o ECT (eletroconvulsoterapia). Em termos de eficácia, em ensaios clí- 
nicos, parece não haver diferenças significativas entre as várias drogas, o que não significa dizer que cada paciente responderá a diferentes antidepressivos da mesma maneira.

\section{Qual é o melhor antidepressivo?}

Esta pergunta deveria ser formulada da seguinte forma: qual o melhor antidepressivo para quem? Todas as classes têm eficácia similar, portanto, a escolha do antidepressivo deve ser baseada nas características da depressão, efeitos colaterais, risco de suicídio, outros distúrbios clínicos, terapia concomitante, tolerabilidade, custo, danos cognitivos, etc.

Deve-se questionar o próprio conceito de "antidepressivo". Afinal, as drogas antidepressivas são utilizadas para muitos outros distúrbios médicos, além da depressão (tabela 1). Infelizmente, na falta de uma definição melhor, continuaremos a usar a supramencionada denominação.

\section{Como os antidepressivos são classificados?}

A classificação mais usada dos antidepressivos tem sido baseada no neurotransmissor/receptor envolvido em seu mecanismo de ação (tabela 2).

\section{Quando os antidepressivos não fazem efeito?}

Dosagens inadequadas e não adesão são as principais causas da falha de resposta. A escolha da droga deve considerar esses fatores. Praticamente todos os antidepressivos podem ser dados uma vez por dia. O modo mais eficiente de tratar a depressão tem sido relacionado com uso de tricíclicos ou inibidores seletivos de recaptação da serotonina (ISRSs).

É evidente, pois permite que as drogas sejam administradas uma vez por dia, com exceção dos tricíclicos que, apesar de meia vida em torno de 24 horas, devido aos efeitos colaterais, são administrados de 2 a 3 vezes por dia.

\section{Tabela 1 - Usos de antidepressivos} em outros distúrbios médicos

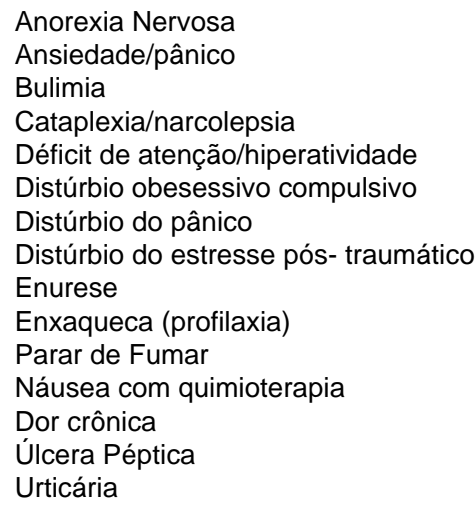

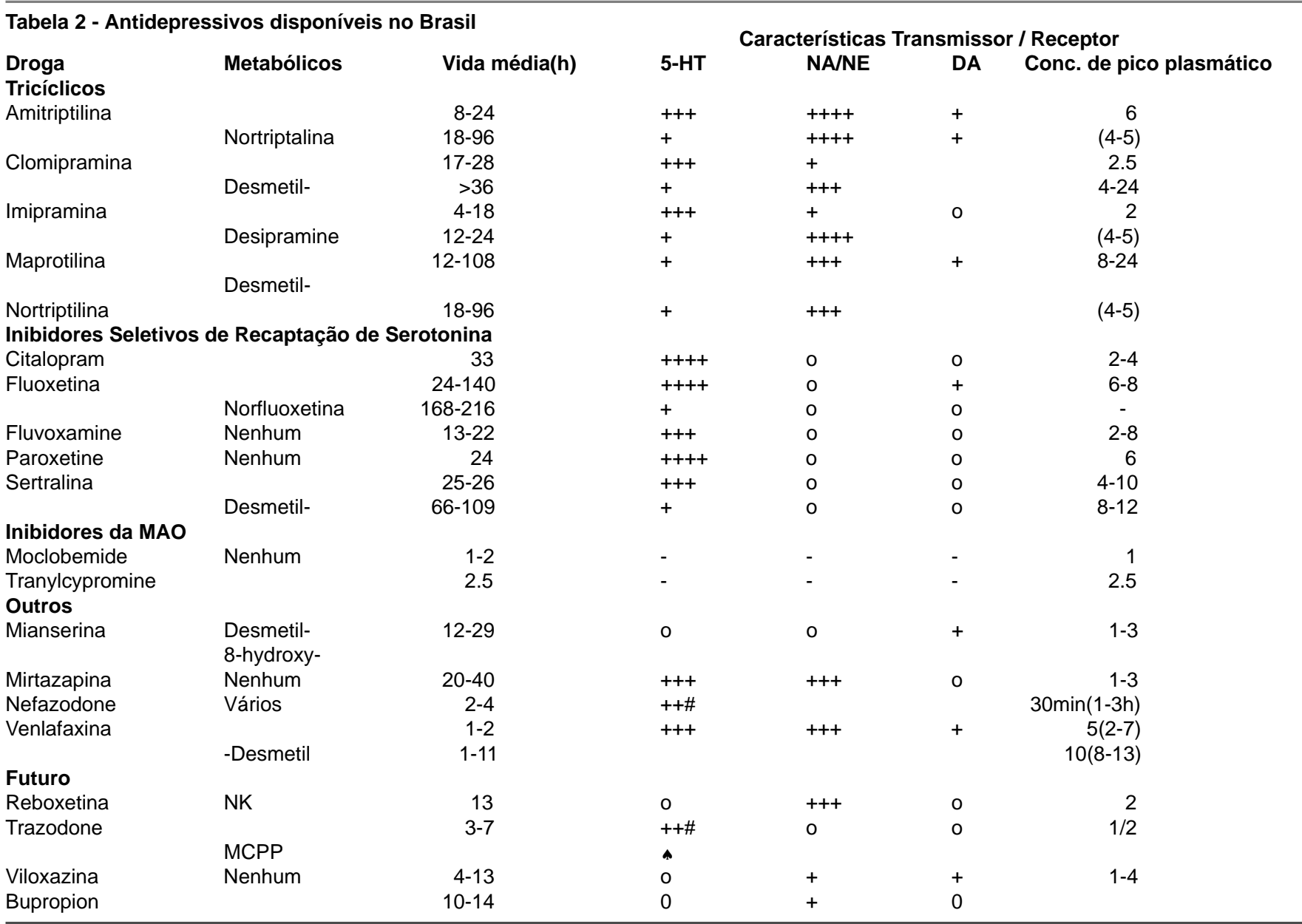


Tabela 3 - Efeitos colaterais dos antidepressivos disponíveis no Brasil

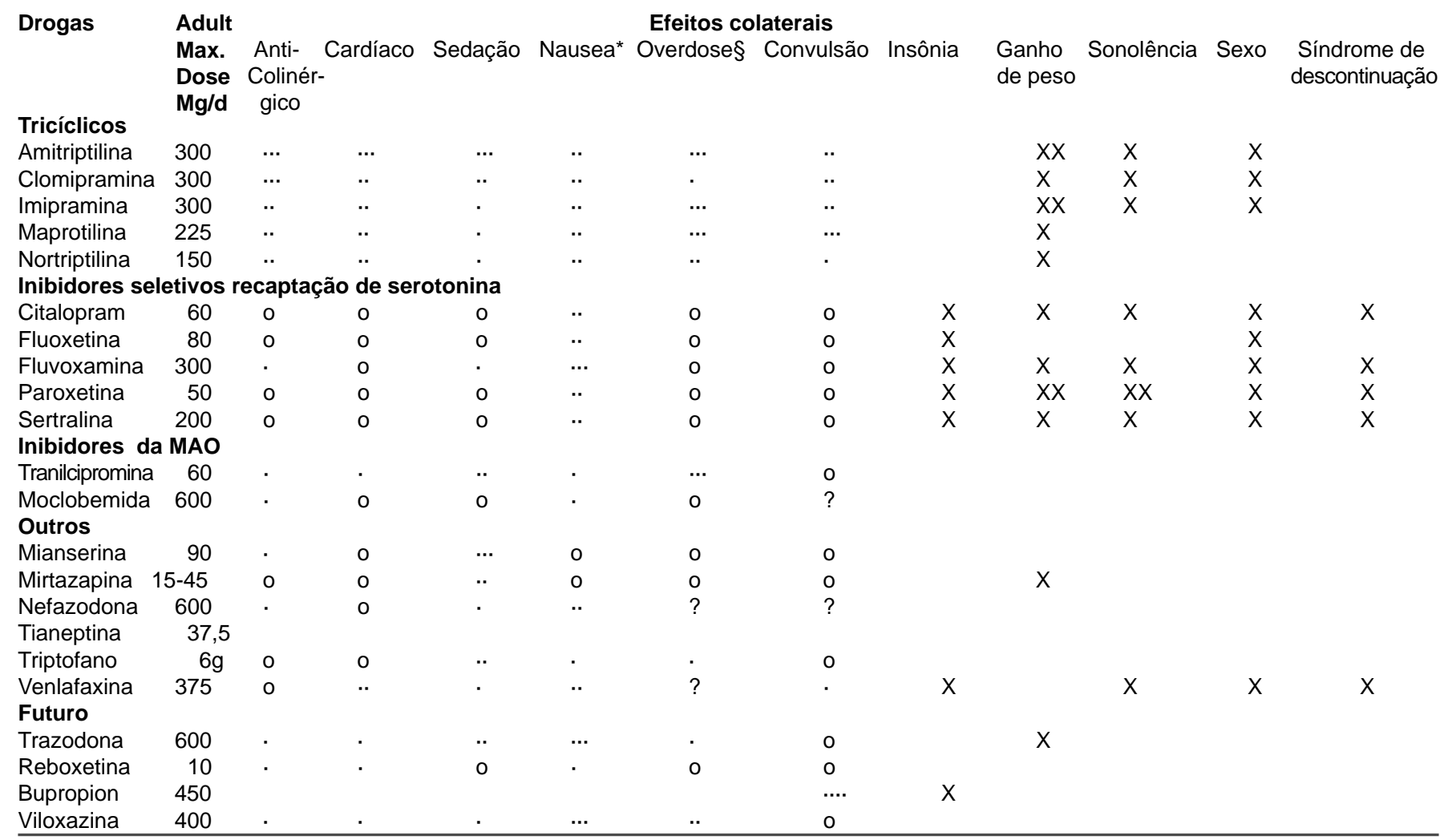

Por que os efeitos colaterais são importantes na escolha dos antidepressivos?

A principal variável relacionada à não adesão dos pacientes são os efeitos colaterais. Portanto, a redução dos efeitos colaterais é fundamental para o êxito do tratamento. Os efeitos mais comuns estão descritos na tabela 3 .

Os principais efeitos colaterais dos tricíclicos são anticolinérgicos. Já com os ISRSs os efeitos sexuais despontam. Neostigmina na dose de $7,5 \mathrm{mg}$ a $15 \mathrm{mg}$, tomada $30 \mathrm{minu}-$ tos antes da relação, tem sido utilizada para aumentar a libido, e a ciproheptadina, $4 \mathrm{mg}$ ao dia por via oral, pode reverter a anorgasmia.

\section{Os antidepressivos tricíclicos são realmente mais baratos?}

O custo inicial dos antidepressivos tricíclicos ainda continua mais baixo do que o de outros antidepressivos. Entretanto, se forem computados os custos totais do tratamento (horas de atendimento, dias perdidos, etc), os antidepressivos mais modernos ficam mais baratos.

\section{Como é dividido o tratamento antidepressivo?}

Usualmente é dividido nas seguintes fases: aguda, continuação (até 6 meses) e preventiva (após 6 meses). Na prática, esta divisão tem importância relativa, visto que a droga com a qual o paciente melhorou deve ser prescrita nas fases subseqüentes do tratamento. A pergunta mais apropriada seria: por quanto tempo deve-se prescrever a medicação?

As doses de continuação devem ser as mesmas ou próximas às doses terapêuticas. ${ }^{18,19}$ Em pacientes idosos, a terapia de continuação até dois anos após a melhora pode ser necessária. ${ }^{20}$ Uma taxa de recaída de até $50 \%$ é observada se o tratamento é inadequado, ou nenhum tratamento após resposta inicial é observado. ${ }^{5}$

\section{Que conduta adotar na terapia aguda?}

$\mathrm{O}$ tratamento adequado da depressão requer não somente a melhora dos sintomas observados na fase aguda, mas também a observação de quais, dos referidos sintomas, não retornam no período de manutenção (recaída), enquanto a pessoa permanece vulnerável. As seguintes variáveis devem ser consideradas:

1. Eliminar as causas contribuintes - causas ambientais, agentes físicos, outras drogas capazes de causar a depressão, excesso de cafeína, etc.

2. Aumentar a dosagem de antidepressivo aos níveis terapêuticos, considerando a resposta e a tolerância do paciente.

3. Doses adequadas, por exemplo, de $150 \mathrm{mg}$ ou mais de tricíclicos, por períodos adequados de tempo. Quatro semanas ou mais são necessárias para um efeito ótimo. ${ }^{21}$ Mesmo assim, $25 \%$ não respondem por seis semanas. ${ }^{22}$

4. Se a depressão continua após quatro semanas de dosagem terapêutica, outro antidepressivo deve ser tentado. ${ }^{21}$

\section{Quando mudar de antidepressivo?}

Mudança de antidepressivo antes de três semanas deve-se à ansiedade da família, do médico, ou dos dois juntos. Os paci- 
entes devem ser orientados para aguardar as três semanas. Um incremento nas sessões psicoterápicas pode ser indicado e, se for necessário, a prescrição de algum ansiolítico.

Após quatro semanas, se o antidepressivo não tiver tido o efeito desejado, uma mudança de classe de antidepressivo e/ou potencialização da terapia podem ser experimentadas. Infelizmente, não há um algoritmo disponível de forma consensual que oriente qual droga deve ser experimentada após a primeira ter falhado no alívio dos sintomas depressivos. ${ }^{21}$

\section{Antidepressivos causam dependência ou síndrome de retirada?}

Os pacientes também devem ser avisados que os antidepressivos não são aditivos, embora síndromes de descontinuação possam ser observadas em alguns pacientes. Síndrome de descontinuação ou retirada tem sido mencionada na literatura, incluindo alterações somáticas e gastrintestinais, alterações do sono, distúrbio dos movimentos, e hipomania. ${ }^{23}$ Sintomas de descontinuação normalmente aparecem de 1 a 14 dias após o fim do tratamento e melhoram dentro de uma semana. Síndrome de descontinuação não deve ser confundida com recorrência de depressão, que começa em médis de 3 a 15 semanas após o término de utilização do antidepressivo e continua a piorar. ${ }^{24}$ Sintomas de retirada podem ocorrer com dosagens não tomadas. Os tratamentos do sintoma de descontinuação incluem: reinstalação de baixas doses do antidepressivo, uso de anticolinérgicos para alívio sintomático ou simplesmente o ato de esperar. ${ }^{25}$

\section{Os antidepressivos devem ser prescritos indefinidamente?}

A Associação Psiquiátrica Americana sugere, pelo menos, 16 a 20 semanas com doses completas após a melhora ou remissão completa. ${ }^{25}$ A Organização Mundial da Saúde sugere 6 meses ou mais após a melhora. ${ }^{26}$ Entre $50 \%$ e $85 \%$ das pessoas sofrendo de um episódio de depressão aguda irão ter um futuro episódio, usualmente dentro de dois a três anos, se não for tratado. Pacientes com um episódio prévio de depressão apresentam um risco 10 vezes maior de recorrência de depressão em relação àqueles indivíduos que não apresentaram nenhum episódio prévio de depressão. O risco é 14 a 18 vezes maior em relação àqueles pacientes que tiveram mais de um episódio prévio de depressão. ${ }^{27}$

Essas recomendações devem ser levadas em consideração, mas também deve-se avaliar a intensidade e freqüência dos episódios antidepressivos atual e anterior(es) antes de tomar uma decisão acerca do tempo de terapia. Assim, um primeiro episódio de um paciente com tentativa de suicídio deve ter um acompanhamento posterior maior e melhor monitorado que um outro paciente em primeiro episódio, mas sem risco de vida. Da mesma maneira, um paciente com um episódio aos 20 anos, o segundo aos 21, e no momento apresenta o terceiro episódio aos 22 necessitaria de um tratamento profilático que outro paciente com um episódio aos 20 anos, outro aos 30 anos, e agora está apresentando o terceiro episódio aos 40 anos.

\section{Como proceder quando o risco de suicídio é detectado?}

Deve-se pesar o sigilo que o paciente suicida algumas vezes nos solicita versus o compromisso terapêutico com a vida que o médico deve ter. O ECT é uma opção importante, pois os antidepressivos levam em torno de quatro semanas para fazer efeito, bem como a vigilância do suporte social deverá ser envolvida se a internação não é possível.

O próprio antidepressivo tem sido associado a tentativas de suicídio - no Reino Unido cerca de 300 pessoas morrem de dosagem de tricíclicos a cada ano. ${ }^{28} \mathrm{O}$ risco de suicídio parece ser o mesmo com tricíclicos e com as novas drogas, mas as taxas de morte são maiores com tricíclicos $-97 \%$ de todas as mortes por superdosagem de antidepressivos foram decorrentes de tricíclicos. ${ }^{29}$

\section{Quais os fatores de risco para a recaída e recorrência?}

Distimia, doenças psiquiátricas não-afetivas, distúrbio médico crônico, história de recaída prévia, tentativas de suicídio, características psicóticas, e severos danos no funcionamento do indivíduo aumentam a probabilidade de agudização do episódio atual (recaída) e de episódios subsequientes (recorrência).

Se o episódio anterior ocorreu há menos de dois anos e meio, o tratamento por pelo menos cinco anos pode ser necessário. ${ }^{1,}$ ${ }^{21}$ Alguns grupos recomendam que a duração do tratamento do $1^{\circ}$ episódio seja de 12 meses após a melhora, $2^{\circ}$ episódio de dois a três anos, e o $3^{\circ}$ episódio de cinco anos ou mais.

\section{Quando interromper a terapia antidepressiva?}

O paciente deve estar a par dos riscos envolvidos. Se ainda assim a descontinuação é processada, seria apropriado ir reduzindo as doses lentamente por um período mínimo de quatro semanas para avaliar se está ocorrendo recaída. ${ }^{30}$

Os níveis de descontinuação dos IRSS são menores (10\%) quando comparados aos dos tricíclicos (20\%).

\section{Como apressar o efeito dos antidepressivos?}

Algumas drogas têm sido propostas no sentido de apressar o efeito dos antidepressivos. Duas alternativas têm sido mencionadas - a utilização do pindolol e a buspirona, ${ }^{4}$ mas com eficácia duvidosa.

\section{Como tratar depressão resistente?}

De $30 \%$ a $80 \%$ dos pacientes deprimidos podem ter recebido doses inadequadas de antidepressivos, e destes, 50\% respondem somente com o aumento da dose. A depressão resistente verdadeira, entretanto, precisa de um enfoque sistemático para melhorá-la. Se a droga utilizada for um tricíclico:

1. usar doses mais altas até 300mg de tricíclicos ou mais, ou até a tolerância;

2. checar os níveis de séricos, 300 a 400 microgramas por litro; ${ }^{31}$ 3. se outro antidepressivo tiver sido usado, verificar se outras classes foram usadas em suas doses máximas;

4. usar combinações lógicas de antidepressivos (lítio e tricíclico), mas evitar carbamazepina com tricíclicos porque os níveis sangüíneos dos antidepressivos são reduzidos. ${ }^{32}$

Como realizar a potencialização da terapia antidepressiva? Duas estratégias têm sido bastante utilizadas na potencialização da terapia antidepressiva. A primeira é usando o lítio e a segunda é através de agentes tireoidianos. ${ }^{14}$ 


\section{Os antidepressivos perdem eficácia?}

Para muitos depressivos, perda da eficácia durante o tratamento de manutenção tem sido relatada em algo entre $9 \%$ e $33 \%$ dos pacientes. As razões podem incluir: falta de adesão, perda da resposta placebo inicial, perda do efeito da droga, tolerância farmacológica, acumulação de metabólicos, mudança na patologia da doença, cicladores rápidos, perda da eficácia profilática. Estratégias para superar este problema incluem: aumento da dose, diminuição da dose de modo que fique na janela terapêutica, adição de bromocriptina, potencialização por estabilizadores do humor e anticonvulsivantes, uso de hormônios tireoidianos, mudança para uma classe diferente de droga e garantia da adesão. ${ }^{5,24}$

\section{O ECT é ultrapassado?}

Definitivamente não. Em depressões graves com risco de suicídio, com características psicóticas e em grávidas, o ECT é uma excelente opção desde que seja administrado de forma ética com anestesia, pessoal treinado e ambiente apropriado. Os principais efeitos do ECT são cognitivos.

\section{Devemos traduzir os ensaios clínicos para a prática psiqui- átrica literalmente?}

Não, porque os pacientes incluídos nos ensaios clínicos com antidepressivos são pacientes virtuais - raramente são vistos em consultórios psiquiátricos, pois só têm depressão, não apresentam qualquer outro distúrbio clínico e/ou psiquiátrico, não tomam qualquer outra medicação, e todos são jovens com idades entre 20 e 40 anos. Os ensaios clínicos nos demonstram a eficácia dos antidepressivos, mas quanto aos estudos sobre eficiência, só a prática clínica continuada nos oferece respaldo.

Deve-se mencionar que a qualidade da relação médico-paciente é fundamental para o sucesso terapêutico do tratamento antidepressivo, pois um terço dos pacientes melhoram em ensaios clínicos com o placebo. Qual a porcentagem dos dois terços que melhoram com o antidepressivo, e têm isto atribuído ao efeito do placebo?

\section{Quais os fatores clínicos que influenciam o tratamento?}

O tratamento antidepressivo torna-se mais complicado quando são observados: risco de suicídio; melancolia; severidade; episódios recorrentes; mania ou hipomania prévia; depressão com características psicóticas; depressão com características catatônicas; depressão com características atípicas; dependência de álcool ou abuso de substâncias; depressão com pânico e outros distúrbios de ansiedade; depressão pós-psicótica; depressão durante a gravidez ou após o parto; depressão superposta à distimia; e depressão superposta a distúrbios de personalidade. ${ }^{1}$

\section{Quais as complicações do tratamento antidepressivo?}

É necessário, antes de iniciar terapia com antidepressivo, investigar a coexistência de distúrbios decorrentes do uso de substâncias e outras condições médicas que estão sendo trata- das concomitantemente, para evitar interações medicamentosas indesejáveis.

Estima-se que a hipomania é uma complicação que atinge de $5 \%$ a $20 \%$ de pacientes deprimidos tratados com antidepressivos.

Efeitos colaterais mais freqüentes como tontura e sedação, alterações cognitivas e comprometimento subclínico da memória podem ser decorrentes de efeitos anticolinérgicos.

Complicações resultantes de bloqueio muscarínico são: boca seca, visão turva, constipação e esforço para urinar.

Ganho de peso é induzido principalmente por antidepressivos tricíclicos, inibidores da monoaminoxidase (IMAO) e lítio.

Perda das funções de ereção e ejaculação no homem e perda da libido e anorgasmia em ambos os sexos são observados principalmente com os ISRSs.

Indução de convulsão pode ser atribuída a alguns antidepressivos - o risco tem sido discreto (menor do que 1\%) e pode aumentar com o aumento da dose.

Antidepressivos tricíclicos, trazodona e inibidores da MAO usualmente provocam hipotensão ortotática como principal efeito cardiovascular. Este efeito indesejável poder ser minimizado com o aumento mais lento da dose.

Distúrbio do sono e ansiedade podem ser exacerbados por fluoxetina em alguns pacientes. Desipramina e bupropion podem também aumentar a ansiedade.

Estes sintomas adversos podem ser contornados com administração de pequenas doses da droga usada. ${ }^{1}$

\section{Quando hospitalizar o indivíduo com depressão?}

Nas seguintes situações: quando o risco de suicídio e homicídio é claro, quando falta ao paciente suporte psicossocial, quando há abuso de substância grave ou o paciente não coopera. ${ }^{1}$

\section{Conclusões}

\section{Existe antidepressivo ideal?}

$\mathrm{O}$ antidepressivo ideal deveria ser eficaz em todas as formas de depressão, inclusive as severas, não ter qualquer efeito colateral, ter custo baixo, poucas interações medicamentosas, poder ser aplicado em todas as idades, melhorar a qualidade do sono, sem distúrbios colaterais, ter posologia fácil e efeito ansiolítico. Obviamente, esta droga ainda não existe (existirá?), de modo que decidir o que prescrever ou não prescrever para qual paciente, por enquanto, é uma questão absolutamente essencial na prática psiquiátrica.

\section{Qual o futuro da terapia antidepressiva?}

Novas drogas estarão disponíveis no mercado em breve. O bupropion ainda não é fabricado no Brasil. A reboxetina já disponível em alguns países ainda não se encontra disponível no Brasil, nem o milnacipran, um novo antidepressivo. Estes poderão ser os novos antidepressivos disponíveis no nosso país.

Deve ser mencionado que a abordagem antidepressiva ainda se situa na sinapse celular. Certamente no próximo milênio, além das abordagens atuais, será possível prever que os antidepressivos da próxima geração atuarão no interior da célula. 


\section{Referências bibliográficas}

1. American Psychiatric Association. Steering committee on practice guidelines. Washington DC: APA Practice Guidelines; 1996.

2. Liskow PA, Perry PJ, Alexander B. Psychotropic drug handbook. In: Antidepressants. 7th ed. Washington, DC: 1997. p. 131-220.

3. Pies RD. Clinical manual of psychiatric diagnosis and treatment: A biopsychosocial approach. 1st ed. Washington DC: 1994.

4. Stahl SM. Psicofarmacologia: Bases neurocientíficas e aplicações clínicas. Rio de Janeiro (RJ): Medsi; 1998.

5. Bazire S. Psychotropic Drug Directory. The professionals' pocket handbook and aide memoire. Wilts (UK): Quay Books; 1999.

6. Potter WZ, Manji HK, Rudorfer MV. Textbook of psychopharmacology. In: Schatzberg AF, Nemeroff CB, editors. Tricyclics and tetracyclics. 2nd ed. Washington DC: The American Psychiatric Press; 1998. p. 199-218.

7. Tollefson GD, Rosenbaum JF. Textbook of psychopharmacology. In: Schatzberg AF, Nemeroff CB, editors. Selective serotonin reuptake inhibitors. 2nd ed. Washington DC: The American Psychiatric Press; 1998. p. 219-37.

8. Krishnan KR. Textbook of psychopharmacology. In: Schatzberg AF, Nemeroff CB, editors. Monoamine oxidase inhibitors. 2nd ed. Washington, DC: The American Psychiatric Press; 1998. p. 239-49.

9. Golden RN, Dawkins K, Nicholas L, Bebchuk JM. Textbook of psychopharmacology. In: Schatzberg AF, Nemeroff CB, editors. Trazodone, nefazodone, bupropion, and mirtazapine. 2nd ed. Washington DC: The American Psychiatric Press; 1998. p. 251-69.

10. Kent JM, Gorman JM. Textbook of psychopharmacology. In: Schatzberg AF, Nemeroff CB, editors. Venlafaxine. 2nd ed. Washington DC: 1998. p. 301-6.

11. Montgomery AS. Psychopharmacology the fourth generation of progress. In: Bloom FE, Kupfer DJ, editors. Selective serotonin reuptake inhibitors in the acute treatment of depression. New York: Raven Press; 1995. p. 1043-51.

12. Burke MJ, Preskorn SH. Psychopharmacology the fourth generation of progress. In: Bloom FE, Kupfer DJ, editors. Short-term treatment of mood disorders with standard antidepressants. New York: Raven Press; 1995. p. 1053-65.

13. Prien RF, Kocsis JH. Psychopharmacology the fourth generation of progress. In: Bloom FE, Kupfer DJ, editors. Long-term treatment of mood disorders. New York: Raven Press; 1995. p. 1067-79.

14. Thase ME, Rush AJ. Psychopharmacology the fourth generation of progress. In: Bloom FE, Kupfer DJ, editors. Treatment-resistant depression. New York: Raven Press; 1995. p. 1081-97.

15. Katz MM, Koslow SH, Maas JW, Frazer A, Bowden CL, Casper R, et al. The timing, specificity and clinical prediction of tricyclic drug effects in depression. Psychol Med 1987; 17:297-309.

16. Quitkin FM, Rabkin JG, Markowitz JM, Stewart JW, McGrath PJ, Harrison W. Use of pattern analysis to identify true drug response. Arch Gen Psychiatry 1987; 44:259-64.

17. Quitkin FM, Rabkin JG, Ross D, McGrath PJ. Chronological milestones to guide drug change. When should clinicians switch antidepressants? 1984; 41:238-45.

18. Frank E, Kupfer DJ, Perel JM, Cornes C, Mallinger AG, Thase ME, et al. Comparison of full-dose versus half-dose pharmacotherapy in the maintenance treatment of recurrent depression. J Aff Dis 1993; 27:139-45.

19. Frank E, Kupfer DJ, Perel JM, Cornes C, Mallinger AG, Thase M, et al. Comparison of full-dose versus half-dose pharmacotherapy in the maintenance treatment of recurrent depression. Arch Gen Psychiatry 1990; 47(12):1093-9.

20. Ardern M, Bergmann K, Conway J, Coolling N, Davies G, Fisk J et al. How long should the elderly. Br J Psychiatry 1993;162:175-82.

21. Katon W, Von Korff M, Lin EB, Simon G, Walker E, Bush T, et al. Collaborative management to achieve depression treatment guidelines. Journal of Clinical Psychiatry 1997; 58 Suppl 1:20-3.

22. Quitkin FM, Rabkin JG, Ross D, McGrath PJ. Duration of antidepressant drug treatment: what is an adequate trial? Arch Gen Psychiatry 1992; 49(10):769-73.

23. Bialos D, Giller E, Peter J, Docherty J, Harkness. Recurrence of depression after discontinuation of long-term amitriptyline treatment. Am J Psych 1982;139:325-29.

24. Coupland NJ, Bell CJ, Potokar EP. Efeitos da descontinuação de inibidores da recaptação da serotonina. J Clin Phychopharmacol 1996;16(5):1-12.

25. American Psychiatric Association: Steering committee on practice guidelines. Practice guideline for major depressives disorder in adults. APA Practice Guidelines. Am J Psych 1993;150(Suppl):1-26.

26. Montgomery SA, Roberts A, Patel AG. Placebo-controlled efficacy of antidepressants in continuation treatment. Int Clin Psychopharmacol 1994; 9 Suppl 1:49-53.

27. Thase ME, Sullivan LR. Relapse and recurrence of depression: A practical approach for prevention. CNS Drugs 1995;4(4):261-77.

28. Jick SS, Dean AD, Jick H. Antidepressants and suicide. British Medical Journal 1995;310:215-8.

29. Henry JA, Alexander CA, Sener EK. Relative mortality from overdose of antidepressants. British Medical Journal 1995;310:221-4.

30. Kupfer DJ, Frank E, Perel JM, Cornes C, Mallinger AG, Thase ME, et al. Five-year outcome for maintenance therapies in recurrent depression. Arch Gen Psychiatry 1992;49: 769-73.

31. Hodgkiss AD, McCarthy PT, Sulke NA, Bridges PK. High dose tertiary amine tricyclic antidepressants in the treatment of severe refractory depression: the central role of plasma concentration situations. Human Psycopharmacol 1995;10(5):407-15.

32. McElroy SL, Keck Jr. PE, Pope Jr. HG, Hudson JI. Valproate in the treatment of bipolar disorder: literature review and clinical guidelines. J Clin Pshychopharmocol 1992;12 Suppl 1:42-52.

Correspondência: Fábio Gomes de Matos e Souza

Rua Manuel Jesuino, 974 - Fortaleza, Ceará. CEP: 60175-270, Tel/fax: +55 85 267-3867 